\title{
The Effects of Conserved Charges in a Nuclear Equation of State
}

\author{
B. Mattos Tavares \\ Instituto de Física, Universidade Federal do Rio de Janeiro, 68528, 21945-970 Rio de Janeiro, RJ, Brasil
}

Received on 15 August, 2003.

\begin{abstract}
We report the present status on the construction of an equation of state (EoS) for the strongly interacting matter which is to be used in the hydrodynamical calculations for the ultra-relativistic heavy ion collisions. In the present version, the conservation of isospin, baryon number and strangeness are taken into account. A preliminary hydrodynamical result for our EoS, using the hydro code SPHERIO, is also shown.
\end{abstract}

\section{Introduction}

If the local thermal equilibrium is attained in relativistic heavy ion reactions, hydrodynamical description may be most adequate for the space-time evolution of the system. It is a very powerful method [1]. Once the initial condition (spatial distribution of 4-velocity field and conserved currents) of the system is specified, the subsequent dynamics is determined uniquely by the EoS of the matter composing the system. Therefore, we expect that a systematic analysis of the experimental observables related to the collective dynamics of the system in terms of the hydrodynamical description will offer the important information on the EoS, namely, the thermal properties of the QGP and hadronic matter. For such purposes, it is essential to provide the equation of state in terms of an efficient computational procedure which supplies all the thermodynamical quantities, temperature, chemical potentials, pressure, etc., as functions of the two independent variables of the hydrodynamical calculation, for example, the baryon number density and entropy density. It is also desirable to establish a method to adapt the EoS easily to the changes of various physical model parameters in order to study the effects of these changes in the hydrodynamical evolution of the system. In this work, a method of construction of the EoS is developped and some results are reported.

\section{The equation of state}

In the NEXUS+SPHERIO program [2], the initial condition is provided by the event generator NEXUS, based on the Gribov-Regge model[3] of hadronic collisions. This program generates, in event-by-event basis, the spatial distribution of the energy-momentum tensor $T^{\mu \nu}$ and the baryon number density $n_{B}$ on the hypersurface $\tau=$ const. Assuming that the system reaches the local thermal equilibrium, we then obtain the initial energy and temperature distribution using the EoS for QGP and relativistic hadronic gas. The subsequent dynamical evolution is followed by the
SPHERIO $^{1}$ code.

Following the works in ref. [5, 6, 7], the present EoS assumes the plasma and hadronic phases as a gas of ideal relativistic quantum particles. It exhibits a first order phase transition between the two states as shown below.

\subsection{Relativistic ideal gas, particle mixture and conserved charges}

For a grand-canonical ensemble of ideal-quantum particles the pressure is given by

$$
P=\frac{\theta g}{(2 \pi)^{3}} \int d^{3} k \ln \left(1+\theta e^{\beta\left(\mu-\epsilon_{k}\right)}\right)
$$

were $\theta=1$ for fermions (and -1 for bosons), $\beta$ is the inverse of temperature, $\mu$ is the chemical potential, $g$ is the degeneracy factor and $\epsilon_{k}=\sqrt{k^{2}+m^{2}}$ is the dispersion relation for the particle where $m$ is the mass of the particle.

The density $n$ and the energy density $e$ can be obtained by the usual thermodynamical relations, $n=\left(\frac{\partial P}{\partial \mu}\right)_{V, T}$, $e=\left(\frac{\partial P}{\partial \beta}\right)_{\lambda}$, where $\lambda$ is the fugacity. The entropy density of the gas can be calculated as $s=\beta(P+e-\mu n)$.

When we include conserved charges such as isospin $\left(3^{\text {rd }}\right.$ component), baryon number and strangeness, the chemical potential [8] must be written as $\mu=B \mu_{B}+S \mu_{S}+T_{3} \mu_{3}$ where $B, S, T_{3}$ are baryon, strangeness and isospin quantum numbers, respectively and $\mu_{B}, \mu_{S}, \mu_{3}$ are the corresponding chemical potentials.

\subsubsection{Gas of Quarks and Gluons}

For simplicity, in our EoS we treat the plasma phase as an ideal gas of relativistic quarks and gluons plus the vacuum pressure $B$.

Considering the mixture of flavors, the total pressure is given by the expression

$$
P_{Q G P}\left(T,\left\{\mu_{i}\right\}\right)=P_{\text {Quarks }}\left(T,\left\{\mu_{i}\right\}\right)+P_{\text {Gluons }}(T)-B
$$

\footnotetext{
${ }^{1}$ SPHERIO (Smoothed Particle Hydrodynamical Evolution of Relativistic HEavy IOn collisions) is a code based in the SPH procedure [4].
} 
Here $P_{\text {Quarks }}$ is already a sum of individual $(\mathrm{u}, \mathrm{d}, \mathrm{s})$ quark pressures (each one given by eq.(1)), B is the bag constant to simulate confinement properties and $\mu_{i}=B_{i} \mu_{B}+S_{i} \mu_{S}+$ $T_{3, i} \mu_{3} ; \mathrm{i}=(\mathrm{u}, \mathrm{d}, \mathrm{s})$. For gluons we have used eq.(1) with $\mu=$ 0 . Analogously energy density is given by:

$$
e_{Q G P}\left(T,\left\{\mu_{i}\right\}\right)=e_{\text {Quarks }}\left(T,\left\{\mu_{i}\right\}\right)+e_{\text {Gluons }}(T)+B
$$

And entropy density for the Plasma is

$$
s_{Q G P}\left(T,\left\{\mu_{i}\right\}\right)=s_{\text {Quarks }}\left(T,\left\{\mu_{i}\right\}\right)+s_{\text {Gluons }}(T)
$$

For the total densities we have only

$$
n_{k}^{Q G P}\left(T,\left\{\mu_{i}\right\}\right)=n_{k}^{\text {Quarks }}\left(T,\left\{\mu_{i}\right\}\right) ; k=B, S, 3
$$

because gluon density is zero.

\subsubsection{Gas of Hadrons}

Basically we treat the hadronic phase as a mixture of gases of quantum relativistic particles, except for the excluded volume effect, like a Van der Waalls hard core correction [6, 7], to fit the densities to the data. In this case, we have:

$$
P^{e x c l}=\sum_{t=1}^{h} P_{t}^{i d}\left(T, \tilde{\mu_{t}}\right) ; \quad \tilde{\mu}_{t} \equiv \mu_{t}-v_{t} P^{e x c l}
$$

where $\mu_{t}=B_{t} \mu_{B}+S_{t} \mu_{S}+T_{3, t} \mu_{3}$ is the chemical potential of the $\mathrm{t}$-th hadron specie, $h$ is the maximum number of hadrons considered and $v_{t}$ is the excluded volume of the t-th hadronic species. $P^{e x c l}$ is determined iteratively. For $n_{k}, e$ and $s$ of the $\mathrm{t}$-th hadronic species $(\mathrm{k}=\mathrm{B}, \mathrm{S}, 3$ as before) we have:

$$
Q_{t}^{e x c l}=\frac{Q_{t}^{i d}}{1+\sum_{i=1}^{h} v_{i} n_{i}^{i d}\left(T, \tilde{\mu}_{i}\right)}
$$

where $Q=n_{k}, e$ or $s$. The superscript $i d$ means ideal, so it should be calculated as in the section 2.1. To get the total $n_{k}, e$ or $s$ of the hadron gas (HG), we must sum over the hadrons. In the present calculation, we took the bag constant $B=380 \mathrm{MeV} / \mathrm{fm}^{3}$ and excluded volume $v_{0}=4 \pi R_{0}^{3} / 3$ for baryons (with $R_{0}=0.7 \mathrm{fm}$ ) and $v_{0}=0$ for mesons, as done in [6]. Other possibilities for excluded volume (e.g ref.[5]) will be explored in future works. All known mesons with mass under $2 \mathrm{GeV}$ and baryons with mass under $2.5 \mathrm{GeV}$ were included in calculations [9]. We took quark masses $m_{u}=1.5 \mathrm{MeV}, m_{d}=3.0 \mathrm{MeV}$ and $m_{s}=120.0$ $\mathrm{MeV}$, although the light quark masses are irrelevant in our EoS.

\subsection{The phase transition}

To construct our phase diagram, we determine the phase boundary using Gibbs equilibrium condition[10] between the QGP and HG phases, i.e. we require thermal, mechanical and chemical equilibrium (for $\mu_{B}$ only) during the phase transition. In the present work, we only consider the case where strangeness and isospin vanishes, thus, the chemical potentials $\mu_{S}$ and $\mu_{3}$ are determined by $\left(T, \mu_{B}\right)$. Some examples of our numerical phase diagrams can be found in Figs. 1 and 2.

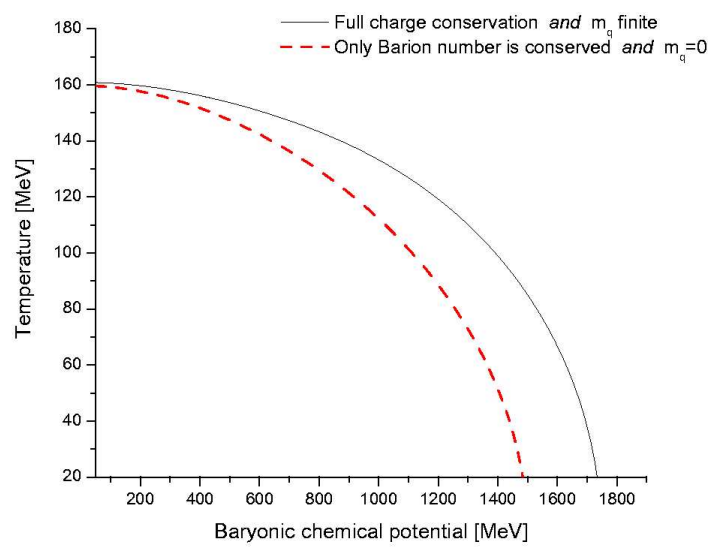

Figure 1. Phase diagrams for $\mathrm{T}$ and $\mu_{B}$.

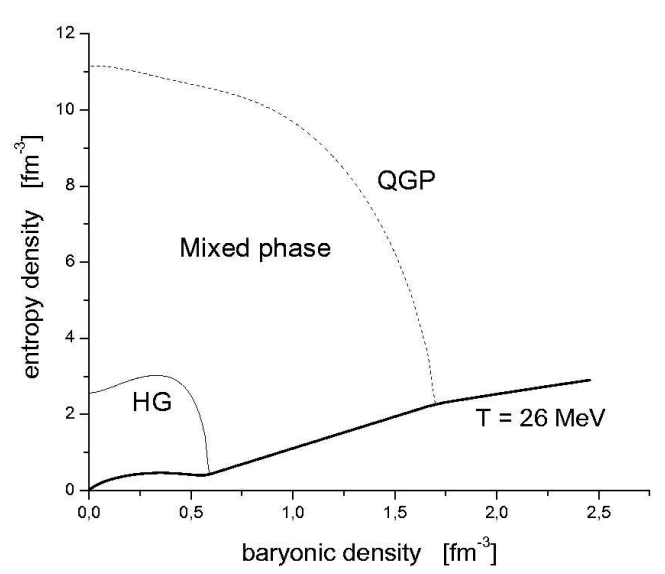

Figure 2. A phase diagram in $\left(s, n_{B}\right)$ plane.

Recent lattice calculations [11] indicate that there is a second order "end point" at non zero $\mu_{B}$, and between this point and $\mu_{B}=0$ the transition should be a smooth cross over[12]. Our simple treatment does not have this feature. It maybe interesting to find a simple parametrization of the equation of state, for instance, in terms of the variation of the bag constant as a function of the temperature and chemical potencial, to simulate the appearance of the critical point.

\section{Numerical procedure and Results}

In order to permit a hydro code to give reliable outputs within a reasonable time of calculation, the numerical EoS must furnish the thermodynamical quantities in a precise and fast way. Specifically in our hydro code SPHERIO, for a given $\left(e, n_{B}\right)$ at the initial condition or $\left(s, n_{B}\right)$ during the dynamical evolution of the system, the EoS need to supply $T, P, s$ or $T, P, e$ respectively. We need also $\mu_{B}, \mu_{S}, \mu_{3}$ at 
the freeze-out time, in order to compute single particle distributions using the Cooper-Frye procedure.

To achieve these purposes, we adopted the following procedure. First, we constructed a set of large numerical tables for the thermal quantities as functions of $T, \mu_{B}$. This has been done evaluating numerically thermal integrals imposing conservation of strangeness and isospin. That is, $\mu_{S}, \mu_{3}$ are determined as functions of $T, \mu_{B}$. These tables are too large to be used directly in the hydro code. Thus, the values of these tables are fitted by domain-wise quadratic functions in $\left(T, \mu_{B}\right)$ and the coefficients of these quadratic functions are stored. In the routine used in hydro code, any output quantity can be obtained by a quadratic interpolation for a given $\operatorname{input}\left(e, n_{B}\right)$ or $\left(s, n_{B}\right)$. The total number of domains in the present version is around $16000(6000$ for hadronic region and 10000 for QGP). For each thermodynamical quantity, we need to store 6 coefficients per domain. We have done a landmark check for these functions, and it took approximately 2 minutes for 500000 points in a rather modest PC (pentium-pro 800Mhz, with $512 \mathrm{MB}$ RAM) which we consider satisfactory.

The quadratic interpolation applied here is very precise and effective, provided that the size of interpolation domain, in the $\left(T, \mu_{B}\right)$ plane, is small enough. In degenerated region, the convergence condition becomes more severe so that some special care should be taken. In the final form of the subroutines we constructed, the error between a given $Q$ and recalculated $Q_{c}$ (where $Q=e, s$ or $n_{B}$ ) using the original thermal integrals is less than $0.01 \%$. In low temperature regions, where the validity of our procedure is not guaranteed, the error can achieve $30 \%$. But we know hydrodynamics rarely achieve these regions, where the hydro procedure becomes to fail. Otherwise a more precise table can easily be constructed.

In the mixed phase we use a linear interpolation between the two points on the phase boundaries of QGP and hadronic gas with the same $T, \mu_{B}, P$. Writing $\alpha$, the fraction of the hadronic component in the mixed phase, any thermal quantity is given as $Q_{\text {int }}=\alpha Q_{H G}+(1-\alpha) Q_{Q G P}$ (where $\left.Q=\left(e, n_{B}, s\right)\right)$. The error in these phase is less than $0.01 \%$ everywhere.

To compute numerically the thermal integrals we have used the Gauss-Laguerre quadrature method [8] which works quite well in most of $T, \mu$ values. However, in extremely degenerate domain, both for fermions and bosons, the method is not adequate. There, the asymptotic formula for the fermions are used instead. For bosons, to avoid the singularity at $(\mu \rightarrow m)$ we modified the singular denominator by an exponentially increasing, but still regular function.

The phase boundaries of the system, necessary for phase judgement of the input point, was computed numerically using Newton method for solving the phase equilibrium equations. Our calculation is good for the temperature higher than $25 \mathrm{MeV}$. Below this temperature, the hadronic gas integrals are not accurate enough to solve the phase equilibrium conditions numerically. Thus, our equation of state is applicable only for $T \geq 26 \mathrm{MeV}$. This will not be a problem since the hydrodynamical procedure is not valid for such low temperatures, as mentioned before.
Analyzing Fig. 1 we note that inclusion of isospin and strangeness conservation enlarges the hadronic domain in $\left(T, \mu_{B}\right)$ plane compared to the equation of state which does not account for these quantum numbers. The inclusion of quark masses does not affect much the form of the phase diagram (only a little in $T_{c}\left(\mu_{B}=0\right)$ ). Change of the bag constant $B$ directly affects the critical temperature at the $\mu_{B}=0$. In Fig. 3 we show the behavior of temperature as function of entropy density for various $n_{B}=$ const, and we can see that it is a continuous function as should be.

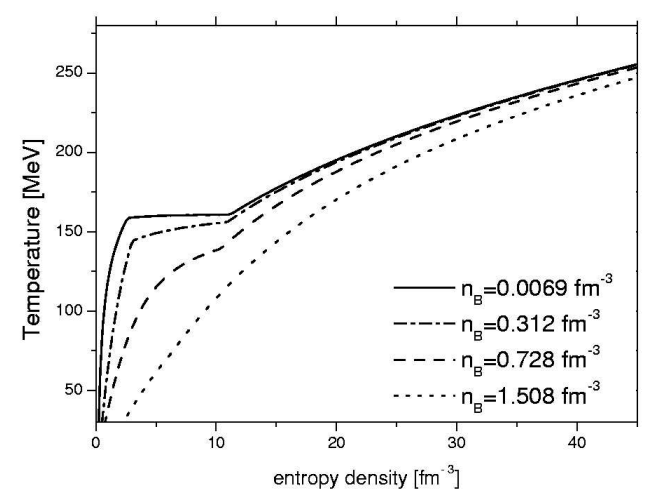

Figure 3. Temperature versus entropy density for various baryon densities.

\section{Conclusions and Perspectives}

In this report, we presented the method of construction of the equation of state used in the hydrodynamical code and a preliminary version of the EoS with strangeness and charge conservation. There are still some points to be improved, but the overall properties are satisfactory. To show how works the present routine of the equation of state, we incorporated it to the SPHERIO code and calculated an example of relativistic nuclear collision. In Fig. 4 we show the result for a rapidity distribution of negative charged hadrons, generated from SPHERIO code, for a single event. The initial condition was given by the NEXUS event generator, corresponding to a $5 \%$ most central $\mathrm{Pb}+\mathrm{Pb}$ collision at energy of $\sqrt{s}=17.3 \mathrm{AMeV}$. The freeze-out temperature was taken to be $140 \mathrm{MeV}$.

Although only one event has been shown, one can see that our result (full line) agrees quite well with the experimental data (dots) from the NA49 collaboration [13] for a $\mathrm{Pb}+\mathrm{Pb}$ collision. Further calculations are in progress, also for BNL-RHIC energies. 


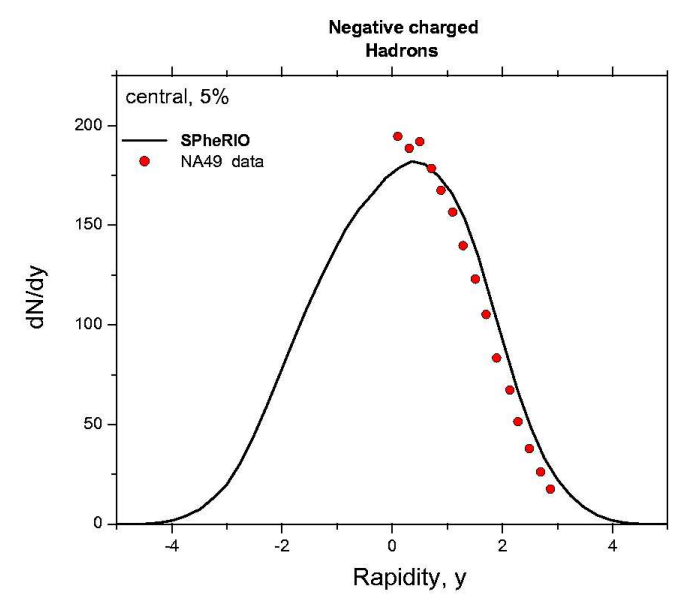

Figure 4. Rapidity distribution for charged hadrons at SPS energies. The dots are experimental data, and the full line is the numerical result.

The present report is a part of the work done in collaboration with Profs. C. E. Aguiar, T. Kodama, Y. Hama, F. Grassi and Dr. O. Scolowski. The author expresses his thanks to Drs. P. Lotti and M. Makler for their kind help. This work is supported by CNPq, FAPERJ, CAPES and FAPESP.

\section{References}

[1] See for example P. Huovinen, nucl-th/0305064; P.F. Kolb and U. Heinz, nucl-th/0305084.

[2] For the union of NEXUS with SPHERIO see, for example, Nucl. Phys A698, 639c (2002).

[3] H.J. Drescher et al, Phys Rev. C 65, 054902 (2002).

[4] For SPH procedure in RHIC see C.E. Aguiar, et al. J. of Phys. G27, 75 (2001).

[5] B. Muzinger et al., Phys. Lett. B 465, 15 (1999).

[6] C.M. Hung, E. Shuryak, Phys. Rev. C 57, 1891 (1997).

[7] G.D. Yen, M.I. Gorenstein, W. Greiner, and S.N. Yang Phys Rev. C 56, 2210 (1997).

[8] T. Kodama, p.3, New states of matter in hadronic interactions, PASI proceedings, AIP (2002).

[9] Particle data, Phys. Rev. D 45, (1992)

[10] J. Sollfrank and U. Heinz, nucl-th/9505004.

[11] Z. Fodor and S.D. Katz, hep-lat/0106002.

[12] K. Rajagopal, hep-ph/0009058.

[13] H. Appelshäuser et al., Phys. Rev. Lett. 82, 2471 (1999). 